\title{
Fetal and Neonatal Responses to Extended Maternal Canine Starvation. II. Fetal and Neonatal Liver Metabolism
}

\author{
E-L. MIETTINEN AND R.M. KLIEGMAN ${ }^{(30)}$ \\ Division of Pediatric Metabolism, Department of Pediatrics, Case Western Reserve University, Cleveland Metropolitan \\ General Hospital, Cleveland, Ohio, USA
}

\begin{abstract}
Summary
The effects of maternal canine starvation (MCS) for 5 days on hepatic intermediates in fetus and fasted neonates were studied at $0,3,6,9$, and $24 \mathrm{~h}$ of age. Maternal starvation reduced the birth weights of the pups by $23 \%(232 \pm 6$ versus $300 \pm 10 \mathrm{~g})$. In the MCS fetuses, hepatic glycogen concentration was significantly decreased (416 versus $526 \mu \mathrm{mole} / \mathrm{g}$ ) whereas uridine diphosphate glucose concentration was significantly increased $(0.196$ versus $0.135 \mu \mathrm{mole} / \mathrm{g}$ ), suggesting diminished glycogen synthetase activity. After birth, hepatic glucose levels were significantly decreased at $3(2.90$ versus $5.95 \mu \mathrm{mole} / \mathrm{g})$ and $24(3.09$ versus 5.29$) \mathrm{h}$ in the MCS pups. Glucose-6-phosphate (G6P) and fructose-6-phosphate (F6P) levels were low in MCS pups throughout the study, G6P being significantly decreased at 9 and $24 \mathrm{~h}$, and F6P at $0,6,9$, and $24 \mathrm{~h}$ in the MCS group. Fructose 1,6-diphosphate did not show any changes among groups. Of greater importance, hepatic ATP concentrations were significantly reduced in the MCS pups throughout the first day of life.

Maternal fasting results in attenuated fetal hepatic glycogen synthesis and an altered transition from fetal to neonatal life. Diminished G6P and F6P levels suggest that hepatic glycolytic activity may be enhanced at the level of phosphofructokinase. In the presence of lactate carbon incorporation into glucose that approaches control rates of gluconeogenesis, the diminished G6P and F6P levels also suggest significant recycling of lactate carbon between glucose and lactate. Furthermore, despite the apparent predominance of glycolysis, diminished hepatic ATP levels suggest that MCS plus neonatal fasting produced a hepatic energy deficiency state. Hepatic substrate deficiency and altered energy production may result from the diversion of glycogen derived glucose to support systemic glucose production.
\end{abstract}

Abbreviations

F6P, fructose-6-phosphate

FDP, fructose 1,6-diphosphate

FFA, free fatty acid

G6P, glucose-6-phosphate

$\alpha-K G$, alpha-ketoglutarate

MCS, maternal canine starvation

PEP, phospholenolpyruvate

UDPG, uridine diphosphate glucose

The effects of maternal starvation on the fetus have been evaluated in rats, sheep, and $\operatorname{dogs}(2,11,14,16,22)$. In the majority of these studies, maternal starvation resulted in a decrease in fetal blood glucose concentration. In the rat and dog, maternal starvation also increased fetal ketone body levels and thus may have provided alternate substrates for fetal oxidation $(11,16)$. In dogs, three days of maternal starvation did not have any effect on fetal hepatic glycogen stores and did not induce gluconeogenesis
$(14,16)$, but did have significant effects on fetal weight and the pup's postnatal metabolic adaptation to birth. Three days of maternal canine starvation resulted in fasting neonatal hypoglycemia and attenuated plasma free fatty acids. Hepatic glycolytic and gluconeogenic intermediate concentrations among these newborn dogs suggested that glycolysis was enhanced whereas gluconeogenesis was attenuated. The latter was hypothesized to be due to decreased hepatic free fatty acid oxidation resulting in diminished cytoplasmic NADH (16).

In order to study the effects of a more significant nutritional deficit on the fetus and its postnatal fasting metabolic adaptation, we have extended the period of maternal canine starvation to 5 days. This study analyzes the effects of extended maternal starvation on hepatic glycolytic and gluconeogenic intermediates and energy and oxidation reduction states in the fetus and fasted newborn pup, during the first day of life. The potential determination of regulatory rate limiting steps may help interpret the changes in circulatory fuels observed in these pups (17). By examining the possible alterations of hepatic metabolic intermediates, an understanding of these altered biochemical events may be assessed employing the standard cross-over plot theory.

\section{MATERIALS AND METHODS}

Materials. All enzymes used were purchased from Sigma, St. Louis, MO.

Animals. Beagle dogs and their pups used in this study were the same as those used in Part I (17).

Experimental design. Cesarean section was done on the canine mother at term (60 \pm 2 days) Immediately at birth and at 3, 6, 9, and $24 \mathrm{~h}$ of age, the pups were sacrificed by guillotine and their livers freeze-clamped at the temperature of liquid nitrogen in less than $7 \mathrm{sec}$. The tissues were stored at $-80^{\circ} \mathrm{C}$ until analyzed. The following intermediates or substrates were analyzed: glycogen, UDPG, glucose, G6P, F6P, FDP, PEP, pyruvate, lactate, citrate, $\alpha-\mathrm{KG}$, malate, triglycerides, alanine aspartate, glutamate, glutamine, ammonia, ATP, ADP, and AMP.

Analyses. The frozen livers were treated and the intermediate concentrations were determined using microfluorometric assays as reported before (16). The interassay variation was between $5-10 \%$ depending on the assay, intra-assay variation being less than $5 \%$. Hepatic glycogen synthase and phosphorylase were determined as per Schwartz and Rall (19).

Statistics. All results were expressed as mean \pm S.E.M. The differences between groups were determined by the Student's $t$ test.

\section{RESULTS}

Extended maternal starvation for 5 days caused a very significant weight loss in the pups. Average birth weight of 32 controls was $300 \pm 10 \mathrm{~g}$ (mean \pm S.E.) and was $232 \pm 6 \mathrm{~g}$ in the 37 offspring of starved mothers $(P<0.0001)$. 
Glycogenolytic intermediates (Table 1). Maternal starvation for 5 days caused a significant reduction of fetal hepatic glycogen levels. At the same time, UDPG concentrations were significantly elevated in the MCS fetuses. Total fetal hepatic glycogen synthase activity was depressed after MCS $(2.65 \pm 0.36$ versus $3.84 \pm 0.45$ nmole $/ \mathrm{mg}$ protein/min, $P<0.05$ ) whereas total glycogen phosphorylase activity was unaffected $(29.29 \pm 4.56$ versus $32.58 \pm$ 7.81).

After birth and throughout the first day of life, the rate of decline in hepatic glycogen concentration was similar in both groups studied so that the absolute values in the MCS group remained lower than in the controls. Neonatal UDPG concentrations stayed constant throughout the first day of life in both groups. Hepatic glucose values did not demonstrate any change during the first day in the control group. In the MCS pups, the intrahepatic glucose concentration declined after birth and was significantly lower than controls at $3 \mathrm{~h}$. At 6 and $9 \mathrm{~h}$, glucose values increased to the control levels but declined again after 24 $\mathrm{h}$ of neonatal fast.

Glycolytic and gluconeogenic intermediates (Table 1). G6P and F6P concentrations in control pups did not show significant developmental changes during the first day of life. The values in the MCS pups were always lower than controls, the differences being significant at 9 and $24 \mathrm{~h}$ for G6P and at $0,6,9$, and $24 \mathrm{~h}$ for F6P. In contrast, FDP levels never demonstrated significant alterations between the groups studied.

Extended maternal starvation did not have an effect on fetal PEP, pyruvate or lactate concentrations. At $3 \mathrm{~h}$, PEP levels were higher than control values in MCS group $(P<0.05)$ and at the same time, lactate levels were lower $(P<0.05)$. Otherwise, there were no differences in PEP, pyruvate or lactate concentrations during the first day in the two groups studied.

Tricarboxylic acid cycle intermediates (Table 1). The intrahepatic levels of citrate, $\alpha-\mathrm{KG}$, and malate were measured. After birth, the values of all these three intermediates increased above fetal levels in both groups. Citrate concentrations always appeared to be lower in the MCS group, but the difference was significant only at $24 \mathrm{~h}$. Alpha-ketoglutarate values in the MCS group showed a similar pattern as those of citrate after birth, and were significantly decreased at 6 and $9 \mathrm{~h}$. Malate concentrations in MCS fetuses were significantly lower than in the fetal controls. After birth, malate levels increased in both groups, reaching the highest value at $3 \mathrm{~h}$ of age in the controls and then began to stabilize. In the MCS group, malate concentrations started to decline after $3 \mathrm{~h}$, and became significantly lower than the controls at 9 and $24 \mathrm{~h}$.

Intrahepatic triglycerides (Fig. I). Intrahepatic triglyceride levels

Table 1. Intrahepatic metabolite concentration in the canine fetus and neonate ( $\mu$ mole $/ g \pm S . E . M$.

\begin{tabular}{|c|c|c|c|c|c|}
\hline & Fetal & $3 \mathrm{~h}$ & $6 \mathrm{~h}$ & $9 \mathrm{~h}$ & $24 \mathrm{~h}$ \\
\hline \multicolumn{6}{|l|}{ Glycogen } \\
\hline Control & $526 \pm 20$ & $508 \pm 27$ & $498 \pm 28$ & $458 \pm 31$ & $305 \pm 50$ \\
\hline $\mathrm{MCS}^{1}$ & $416 \pm 48^{2}$ & $421 \pm 43$ & $399 \pm 45^{2}$ & $371 \pm 38$ & $197 \pm 78$ \\
\hline \multicolumn{6}{|l|}{ UDPG } \\
\hline Control & $0.135 \pm 0.016$ & $0.121 \pm 0.015$ & $0.119 \pm 0.005$ & $0.159 \pm 0.015$ & $0.125 \pm 0.007$ \\
\hline MCS & $0.196 \pm 0.022^{2}$ & $0.121 \pm 0.018$ & $0.131 \pm 0.018$ & $0.152 \pm 0.015$ & $0.113 \pm 0.019$ \\
\hline \multicolumn{6}{|l|}{ Glucose } \\
\hline Control & $4.74 \pm 0.457$ & $5.97 \pm 0.797$ & $5.35 \pm 0.633$ & $5.49 \pm 0.649$ & $5.29 \pm 0.422$ \\
\hline MCS & $3.58 \pm 0.633$ & $2.90 \pm 0.390^{3}$ & $4.22 \pm 0.583$ & $5.59 \pm 1.13$ & $3.09 \pm 0.356^{2}$ \\
\hline \multicolumn{6}{|l|}{ G6P } \\
\hline Control & $0.227 \pm 0.080$ & $0.212 \pm 0.054$ & $0.231 \pm 0.045$ & $0.211 \pm 0.036$ & $0.147 \pm 0.016$ \\
\hline MCS & $0.141 \pm 0.037$ & $0.107 \pm 0.010$ & $0.148 \pm 0.021$ & $0.121 \pm 0.025^{2}$ & $0.073 \pm 0.012^{3}$ \\
\hline \multicolumn{6}{|c|}{$0.121-0.020$} \\
\hline Control & $0.056 \pm 0.011$ & $0.045 \pm 0.009$ & $0.050 \pm 0.008$ & $0.054 \pm 0.010$ & $0.048 \pm 0.003$ \\
\hline MCS & $0.027 \pm 0.006^{2}$ & $0.027 \pm 0.003$ & $0.030 \pm 0.004^{2}$ & $0.018 \pm 0.007^{3}$ & $0.022 \pm 0.006^{3}$ \\
\hline \multicolumn{6}{|l|}{ FDP } \\
\hline Control & $0.019 \pm 0.003$ & $0.016 \pm 0.001$ & $0.017 \pm 0.003$ & $0.018 \pm 0.002$ & $0.020 \pm 0.003$ \\
\hline MCS & $0.016 \pm 0.002$ & $0.015 \pm 0.003$ & $0.018 \pm 0.001$ & $0.015 \pm 0.002$ & $0.013 \pm 0.003$ \\
\hline \multicolumn{6}{|l|}{ PEP } \\
\hline Control & $0.125 \pm 0.020$ & $0.098 \pm 0.013$ & $0.121 \pm 0.015$ & $0.116 \pm 0.021$ & $0.116 \pm 0.021$ \\
\hline MCS & $0.119 \pm 0.011$ & $0.148 \pm 0.015^{2}$ & $0.111 \pm 0.010$ & $0.098 \pm 0.018$ & $0.142 \pm 0.027$ \\
\hline \multicolumn{6}{|l|}{ Pyruvate } \\
\hline Control & $0.123 \pm 0.012$ & $0.090 \pm 0.006$ & $0.075 \pm 0.010$ & $0.082 \pm 0.008$ & $0.073 \pm 0.188$ \\
\hline MCS & $0.121 \pm 0.018$ & $0.090 \pm 0.003$ & $0.077 \pm 0.008$ & $0.092 \pm 0.011$ & $0.066 \pm 0.006$ \\
\hline \multicolumn{6}{|l|}{ Lactate } \\
\hline Control & $2.73 \pm 0.604$ & $1.91 \pm 0.266$ & $1.48 \pm 0.326$ & $1.68 \pm 0.249$ & $1.17 \pm 0.188$ \\
\hline MCS & $2.22 \pm 0.392$ & $1.35 \pm 0.135^{2}$ & $1.42 \pm 0.088$ & $1.55 \pm 0.396$ & $1.39 \pm 0.369$ \\
\hline \multicolumn{6}{|l|}{ Citrate } \\
\hline Control & $0.179 \pm 0.025$ & $0.478 \pm 0.037$ & $0.435 \pm 0.011$ & $0.442 \pm 0.018$ & $0.454 \pm 0.008$ \\
\hline MCS & $0.171 \pm 0.025$ & $0.399 \pm 0.033$ & $0.418 \pm 0.045$ & $0.337 \pm 0.051$ & $0.334 \pm 0.032^{3}$ \\
\hline \multicolumn{6}{|c|}{$\alpha$-Ketoglutarate } \\
\hline Control & $0.093 \pm 0.013$ & $0.165 \pm 0.023$ & $0.168 \pm 0.019$ & $0.187 \pm 0.013$ & $0.132 \pm 0.020$ \\
\hline MCS & $0.104 \pm 0.018$ & $0.129 \pm 0.012$ & $0.093 \pm 0.011^{3}$ & $0.075 \pm 0.011^{4}$ & $0.093 \pm 0.009$ \\
\hline \multicolumn{6}{|l|}{ Malate } \\
\hline Control & $0.575 \pm 0.095$ & $0.948 \pm 0.048$ & $0.663 \pm 0.053$ & $0.721 \pm 0.097$ & $0.733 \pm 0.086$ \\
\hline MCS & $0.376 \pm 0.055^{2}$ & $0.768 \pm 0.138$ & $0.665 \pm 0.123$ & $0.459 \pm 0.069^{2}$ & $0.413 \pm 0.061^{3}$ \\
\hline \multicolumn{6}{|c|}{ Number studied } \\
\hline Control & 6 & 6 & 6 & 6 & 6 \\
\hline MCS & 8 & 9 & 8 & 5 & 6 \\
\hline
\end{tabular}

\footnotetext{
${ }^{1} \mathrm{MCS}$, prior extended maternal canine starvation

${ }^{2} P<0.05$.

$2 P<0.01$.

${ }^{4} P<0.001$.
} 
increased after birth in both groups studied. The values continued to increase until 6-9 $\mathrm{h}$ of age, and then demonstrated a small decline being evident at $24 \mathrm{~h}$. There were no significant differences between the groups studied.

Intrahepatic amino acids (Table 2). Intrahepatic levels of important gluconeogenic precursors, alanine, aspartate, glutamate, and glutamine in addition to ammonia were investigated. There was a significant decline in the alanine concentration after birth in both groups studied; thereafter alanine values remained at this level. Aspartate concentrations did not demonstrate any alterations in controls during the first day. At $9 \mathrm{~h}$, the aspartate concentration in the MCS pups was significantly higher than in the control pups. Hepatic glutamate and glutamine concentrations did not show any changes between the groups. In the MCS pups glutamate levels increased significantly $(P<0.001)$ after birth, compared with controls.

Of more interest were the hepatic ammonia levels. In the fetuses of starved mothers, ammonia concentration was significantly elevated and declined to control values $3 \mathrm{~h}$ after birth. Ammonia

\section{LIVER TRIGLYCERIDES}

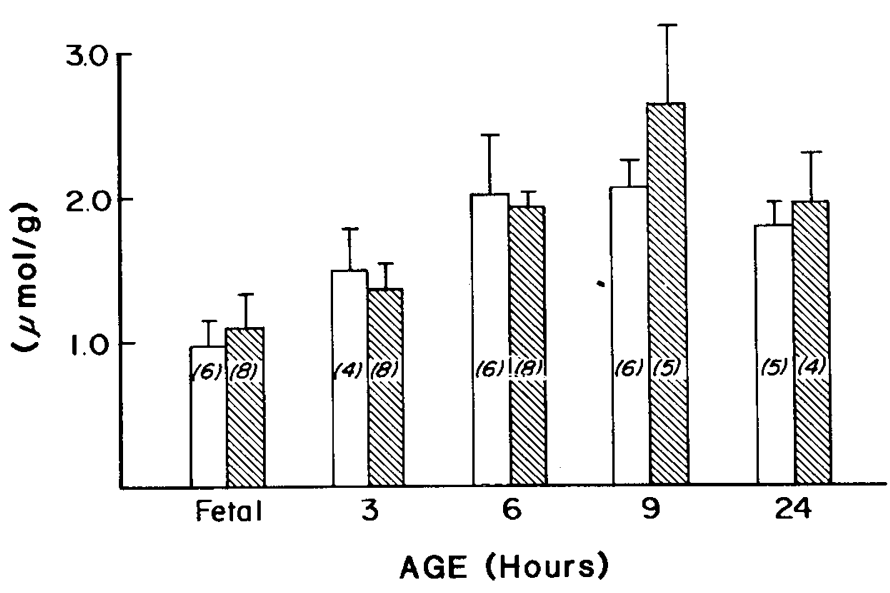

Fig. 1. Fetal and neonatal hepatic triglyceride concentrations (mean \pm S.E.M). Control pups, $\square$; prior extended maternal starvation, पITIV; and number of pups, ( ). levels thereafter began to increase again and were significantly higher than the timed controls at 6, 9, and $24 \mathrm{~h}$.

Intrahepatic energy state (Table 3). The effects of extended maternal starvation upon the hepatic energy state in the fetus and newborn were evaluated by measuring intrahepatic ATP, ADP, and AMP concentrations. Maternal starvation for 5 days did not change fetal ATP, ADP, or AMP concentrations; however, after birth hepatic ATP levels in the control group increased whereas ATP values in the MCS pups remained unchanged and thus were significantly lower throughout the first day of life. There were no changes in the ADP levels during the $24 \mathrm{~h}$ of life or between the groups. Hepatic AMP concentrations began to decline after birth in the control group and stabilized at $6 \mathrm{~h}$ of age. In the MCS group in contrast, there was no noticeable decrease in the AMP values between $0-3 \mathrm{~h}$. After $6 \mathrm{~h}$ of life, however, the AMP levels started to decline to control levels. Hepatic energy charge calculated from ATP, ADP, and AMP according to Atkinson and Walton (1) were not affected at $0-3 \mathrm{~h}$, but were significantly lower than control values at $6-9 \mathrm{~h}$ of age.

Cytoplasmic and mitochondrial oxidation reduction states were analyzed by calculating the NAD/NADH ratios (Table 3 ). The cytoplasmic ratio was calculated from hepatic lactate and pyruvate concentrations and the equilibrium constant for the lactate dehydrogenase reaction according to Williamson et al. (26). Mitochondrial NAD/NADH ratio was determined from hepatic ammonia, $\alpha$-ketoglutarate, and glutamate concentrations. The equilibrium constant for glutamate dehyrogenase was from Williams et al. (26). The cytoplasmic NAD/NADH ratio did not show any changes in the control pups during the first day of life. In the pups of starved mothers, however, the ratio was significantly increased at $3 \mathrm{~h}$ of life reflecting a more oxidized cytoplasmic state. Later on during the first day of life, there was no difference between the groups in the cytoplasmic oxidation-reduction state. The mitochondrial NAD/NADH ratio was significantly elevated in the fetuses of starved mothers, but declined to control levels after birth, and thereafter remained constant throughout the $24 \mathrm{~h}$. At 9 $\mathrm{h}$ of life, the mitochondrial oxidation-reduction state in the control pups was temporarily increased resulting in a significant difference in the NAD/NADH ratio between the two groups.

\section{DISCUSSION}

Glycogen cycle. The fetuses of starved mothers at the time of delivery had significantly decreased hepatic glycogen stores compared to control fetuses. At the same time hepatic UDPG values

Table 2. Intrahepatic amino acid levels in canine fetus and newborn ( $\mu$ mole $/ g \pm$ S.E.M.)

\begin{tabular}{|c|c|c|c|c|c|}
\hline & Fetus & $3 \mathrm{~h}$ & $6 \mathrm{~h}$ & $9 \mathrm{~h}$ & $24 \mathrm{~h}$ \\
\hline \multicolumn{6}{|l|}{ Alanine } \\
\hline Control & $0.976 \pm 0.071$ & $0.510 \pm 0.059$ & $0.383 \pm 0.037$ & $0.400 \pm 0.011$ & $0.536 \pm 0.100$ \\
\hline $\mathrm{MCS}^{1}$ & $0.923 \pm 0.083$ & $0.474 \pm 0.086$ & $0.435 \pm 0.052$ & $0.638 \pm 0.131$ & $0.479 \pm 0.057$ \\
\hline \multicolumn{6}{|l|}{ Aspartate } \\
\hline Control & $0.608 \pm 0.053$ & $0.442 \pm 0.023$ & $0.374 \pm 0.046$ & $0.421 \pm 0.016$ & $0.379 \pm 0.099$ \\
\hline MCS & $0.643 \pm 0.053$ & $0.537 \pm 0.089$ & $0.493 \pm 0.065$ & $0.684 \pm 0.076^{2}$ & $0.534 \pm 0.047$ \\
\hline \multicolumn{6}{|l|}{ Glutamate } \\
\hline Control & $4.54 \pm 0.505$ & $5.33 \pm 0.562$ & $4.88 \pm 0.725$ & $4.58 \pm 0.478$ & $5.62 \pm 0.249$ \\
\hline MCS & $3.86 \pm 0.202$ & $6.41 \pm 0.470$ & $5.20 \pm 0.692$ & $5.47 \pm 0.445$ & $5.25 \pm 0.674$ \\
\hline \multicolumn{6}{|l|}{ Glutamine } \\
\hline Control & $9.02 \pm 0.568$ & $7.45 \pm 0.690$ & $7.21 \pm 0.535$ & $8.08 \pm 0.687$ & $7.15 \pm 0.494$ \\
\hline MCS & $8.07 \pm 0.572$ & $8.33 \pm 0.701$ & $7.17 \pm 0.747$ & $7.39 \pm 0.722$ & $7.36 \pm 1.04$ \\
\hline \multicolumn{6}{|l|}{$\mathrm{NH}_{4}^{+}$} \\
\hline Control & $0.442 \pm 0.050$ & $0.370 \pm 0.058$ & $0.313 \pm 0.066$ & $0.442 \pm 0.064$ & $0.438 \pm 0.071$ \\
\hline $\mathrm{MCS}$ & $0.822 \pm 0.173^{2}$ & $0.466 \pm 0.069$ & $0.502 \pm 0.051^{2}$ & $0.630 \pm 0.083^{2}$ & $0.674 \pm 0.062^{2}$ \\
\hline \multicolumn{6}{|c|}{ Number studied } \\
\hline Control & 6 & 6 & 6 & 6 & 6 \\
\hline MCS & 8 & 9 & 8 & 5 & 6 \\
\hline
\end{tabular}

${ }^{1}$ MCS, prior extended maternal canine starvation.

${ }^{2} P<0.05$. 
Table 3. Intrahepatic energy and oxidation-reduction state in the canine fetus and newborn (mean \pm S.E.M.)

\begin{tabular}{|c|c|c|c|c|c|}
\hline & Fetus & $3 \mathrm{~h}$ & $6 \mathrm{~h}$ & $9 \mathrm{~h}$ & $24 \mathrm{~h}$ \\
\hline \multicolumn{6}{|c|}{ ATP $(\mu$ mole $/ \mathrm{g})$} \\
\hline Control & $1.58 \pm 0.110$ & $1.72 \pm 0.135$ & $2.00 \pm 0.097$ & $2.24 \pm 0.091$ & $1.93 \pm 0.281$ \\
\hline $\mathrm{MCS}^{1}$ & $1.42 \pm 0.140$ & $1.41 \pm 0.094^{2}$ & $1.54 \pm 0.116^{3}$ & $1.57 \pm 0.151^{3}$ & $1.32 \pm 0.060^{2}$ \\
\hline \multicolumn{6}{|c|}{ ADP $(\mu$ mole $/ g)$} \\
\hline Control & $1.27 \pm 0.104$ & $1.14 \pm 0.044$ & $1.06 \pm 0.103$ & $1.11 \pm 0.079$ & $1.37 \pm 0.108$ \\
\hline MCS & $1.06 \pm 0.086$ & $1.00 \pm 0.075$ & $1.11 \pm 0.042$ & $1.10 \pm 0.091$ & $1.28 \pm 0.157$ \\
\hline \multicolumn{6}{|c|}{ AMP $(\mu \mathrm{mole} / \mathrm{g})$} \\
\hline Control & $0.283 \pm 0.033$ & $0.240 \pm 0.035$ & $0.198 \pm 0.022$ & $0.188 \pm 0.025$ & $0.229 \pm 0.020$ \\
\hline MCS & $0.251 \pm 0.030$ & $0.249 \pm 0.025$ & $0.280 \pm 0.026^{2}$ & $0.224 \pm 0.040$ & $0.182 \pm 0.033$ \\
\hline \multicolumn{6}{|c|}{ Energy charge $^{4}$} \\
\hline Control & $0.697 \pm 0.0 \mathrm{i} 0$ & $0.737 \pm 0.020$ & $0.779 \pm 0.018$ & $0.790 \pm 0.014$ & $0.734 \pm 0.026$ \\
\hline MCS & $0.710 \pm 0.025$ & $0.718 \pm 0.013$ & $0.711 \pm 0.016^{3}$ & $0.731 \pm 0.026^{2}$ & $0.705 \pm 0.009$ \\
\hline \multicolumn{6}{|c|}{ NAD/NADH (cytoplasm) ${ }^{5}$} \\
\hline Control & $493 \pm 96.6$ & $445 \pm 35.1$ & $568 \pm 116$ & $493 \pm 75.9$ & $669 \pm 193$ \\
\hline MCS & $576 \pm 104$ & $674 \pm 93.8^{2}$ & $498 \pm 53$ & $618 \pm 109$ & $560 \pm 124$ \\
\hline \multicolumn{6}{|c|}{ NAD/NADH (mitochondrial) ${ }^{6}$} \\
\hline Control & $2.49 \pm 0.451$ & $3.09 \pm 0.718$ & $2.95 \pm 0.681$ & $4.11 \pm 0.829$ & $2.56 \pm 0.380$ \\
\hline MCS & $5.68 \pm 1.28^{2}$ & $2.52 \pm 0.457$ & $2.67 \pm 0.559$ & $2.28 \pm 0.478^{2}$ & $3.30 \pm 0.679$ \\
\hline \multicolumn{6}{|c|}{ Number studied } \\
\hline Control & 6 & 6 & 6 & 6 & 6 \\
\hline MCS & 8 & 9 & 8 & 5 & 6 \\
\hline
\end{tabular}

${ }^{1} \mathrm{MCS}$, prior extended maternal canine starvation.

${ }^{2} P<0.05$.

${ }^{3} P<0.01$.

${ }^{4}$ Energy Charge, $\left.0.5(2 \mathrm{ATP}+\mathrm{ADP}) / \mathrm{ATP}+\mathrm{ADP}+\mathrm{AMP}\right)$.

${ }^{5}$ Cytoplasmic NAD/NADH, $1 / \mathrm{K}_{\mathrm{LDH}} \times$ (pyruvate) $/\left(\right.$ lactate) $; \mathrm{K}_{\mathrm{LDH}}=1.11 \times 10^{-4}$

${ }^{6}$ Mitochondrial NAD/NADH, $1 / \mathrm{Kg}_{\mathrm{DH}} \times\left[(\alpha-\mathrm{Kg}) \times\left(\mathrm{NH}_{4}{ }^{+}\right) /(\right.$glutamate $\left.)\right] ; \mathrm{Kg}_{\mathrm{DH}}=3.87 \times 10^{-3}$.

were significantly increased. It has been shown that UDPG concentrations may be elevated when glycogen synthetase becomes inactive $(6,23)$. This may be supported by the diminished total glycogen synthase levels recorded in the MCS fetal pups. These observations suggest that fetal glycogen synthesis had diminished due to attenuated maternal and probably fetal substrate availability. Diminished provision of maternally derived precursors for fetal glycogen synthesis may have been responsible for this effect. Similar observations have been made in starved fetal rats by Girard et al. (11). Despite the significant depression of fetal hepatic glycogen content there apparently was sufficient glycogen present to support systemic glucose production during the immediate neonatal period when gluconeogenesis was less active (17). In addition, MCS had no effect on postneonatal glycogenolysis, as there were no differences in the rate of hepatic glycogen breakdown between the two groups after birth.

At $3 \mathrm{~h}$ of age, both circulating glucose and hepatic glucose concentrations were significantly decreased in the MCS pups (17). According to Hue and Hers (12), low ambient glucose concentrations arrest glycogen synthesis and activate glycogen phosphorylase in adult rats. Gilbert and Bourbon (9) have shown in fetal rat livers that phosphorylase $a$ activity was elevated until the blood glucose reached values over $5.5 \mathrm{mmole} / \mathrm{liter}$. In spite of low blood glucose concentrations at $3 \mathrm{~h}$ of age in our MCS group, hepatic glycogenolysis was not accelerated above that noted in the controls. This may result from the relationship between blood glucose values and activation of neonatal hepatic glycogen phosphorylase $a$ as described above. Kawai and Arinz (13) have shown a delay in the initiation of glycogenolysis in normal neonatal guinea pigs because of decreased glucagon and epinephrine sensitivity immediately after birth. Hormonal sensitivity is likely to be of the same magnitude in both control and MCS pups $(10,18)$, although this sensitivity in the experimental pups has not been quantified.

Glycolysis and gluconeogenesis. In the liver, the majority of G6P generated from glycogenolysis is either converted to glucose or enters the glycolytic pathway. In mammalian fetuses, increased hepatic G6P levels are said to favor glycogen synthesis because G6P dependent glycogen synthetase is the active enzyme during fetal life $(12,19)$. In our study, both G6P and F6P were low in the
MCS fetuses, thus further suggesting inactivation of glycogen synthesis. After birth, G6P and F6P values (which are in dynamic equilibrium) remained low in the pups of 5-day starved mothers throughout the 24-h study period. Conversion of F6P to FDP requires phosphofructokinase (PFK), an enzyme which potentially exists in an active (dephosphorylated) and a less active (phosphorylated) form (4). Additional regulation of this enzyme by ATP, citrate, FFA, and NADH is important as they are allosteric negative effectors of the enzyme, whereas AMP is a positive effector $(4,24)$. In the present study, hepatic FDP concentrations were similar in both groups whereas ATP and citrate concentrations were lower throughout the neonatal study period in the MCS pups. Estimated hepatic cytoplasmic NADH values were low at 3 $h$ of age. These changes of the allosteric regulators favor increased hepatic PFK activity in MCS fasted pups relative to the control pups. In addition, the diminished F6P concentrations observed after MCS would also suggest enhanced PFK activity. The overall hepatic response may then be a predominance of glycolytic flux, again relative to time-controlled normal pups. In our earlier study, neonatal hepatic glycolysis was also thought to be activated after 3 days of maternal starvation (16).

An additional mechanism acting on G6P and F6P concentrations may be related to the glucose-glucose-6-phosphate futile cycle (12). Diminished G6P and F6P may result from the utilization of these intermediate's carbons for hepatic glucose output by way of a relative activation of G6P compared to hexokinase. Particularly at $3 \mathrm{~h}$ of age when hepatic and circulating levels of glucose are lower than controls, glucose 6-phosphatase activity may deplete these two intermediates (which are in dynamic equilibrium) to support hepatic glucose production (12). Thereafter, throughout the remaining period of neonatal fasting, the combination of hepatic glucose production, enhanced glycolytic pathway activity, and the potential for accelerated recycling of lactate through gluconeogenic pathways may result in the observed metabolic perturbations. The latter event of accelerated recycling of lactate carbon between glucose-lactate and back to glucose is suggested because rates of lactate carbon incorporation into glucose in MCS pups were equivalent to those of controls. In the presence of stimulated phosphofructokinase activity, one would 
have to postulate true futile recycling of this lactate carbon in MCS pups $(4,12)$.

Pyruvate reflects the balance between glycolysis, lactate and pyruvate oxidation and gluconeogenesis. The first step for the oxidation of pyruvate is catalyzed by pyruvate dehydrogenase complex. PDH activity is inhibited by elevated concentrations of $\beta$-OH-butyrate, FFA, and their acetyl-CoA derivatives through acetyl-CoA and NADH (25). Fetuses of 5-day starved mothers had elevated plasma ketone body concentrations, which may have caused inactivation of pyruvate dehydrogenase complex and activation of pyruvate carboxylase. Possible fetal oxidation of ketone bodies or FFA may provide sufficient acetyl-CoA to maintain the activity of the tricarboxylic acid cycle (21). After birth, glycolytically produced pyruvate may have become the major source for acetyl-CoA in MCS pups as plasma ketone body levels now became very low whereas plasma FFA concentrations were temporally lower than in controls (17). The same alterations were noticed in the newborns after 3 days of maternal starvation (16).

Fat metabolism. It has been shown in rats and rabbits that FFA and ketone bodies transported from the mother can serve as precursors for fetal hepatic triglycerides $(5,20)$. After birth, FFA released from the adipose tissue is one source of hepatic triglyceride synthesis (5). In the present study there was a continuous increase in hepatic triglyceride levels until $9 \mathrm{~h}$ in both groups studied. In spite of lower plasma FFA and glycerol concentrations in the MCS pups and the diminished hepatic ATP levels (17), hepatic triglyceride levels increased paradoxically. Low plasma FFA and glycerol concentrations after birth (17) suggest decreased lipolysis or increased utilization of these substrates. Additionally, decreased hepatic ATP, and cytoplasmic NADH levels may indicate that FFA utilization by the liver may have been directed to triglyceride synthesis rather than utilized to support the apparent inadequate rates of oxidative metabolism in fasted MCS pups (3). The decreased appropriate utilization of FFA or glycerol may obligate that hepatic glycolysis be the predominant energy producing pathway as pyruvate rather than fat oxidation is required for ATP production.

Amino acids. Extended maternal starvation did not effect the hepatic amino acid levels studied; however, ammonia concentrations in these livers were significantly elevated at $0,6,9$, and $24 \mathrm{~h}$. Hepatic ammonia formed in amino acid deamination reactions is usually used for ureogenesis. This pathway requires ATP utilization. After birth, hepatic ATP levels were low throughout the first day in the MCS pups. Lack of appropriate energy production combined with normal or increased amino acid utilization (deamination) may explain the increased ammonia levels in the livers of pups after 5 days of maternal starvation.

Energy state. An important and consistent observation among fasted newborn pups subjected to prior maternal starvation was the alteration of neonatal hepatic ATP levels. Extended maternal starvation did not effect fetal hepatic adenine nucleotide concentrations or the energy charge. Hepatic mitochondria were more oxidized in the MCS fetuses than in control fetuses according to calculated NAD/NADH ratios. It has been shown in the guinea pig and rabbit liver that the mitochondrial oxidation-reduction state shifts toward oxidation during fasting $(8,15)$. In our study, the fetuses did not receive sufficient amounts of nutrients from the mother during 5 days of starvation and were also in a fasted state. After birth, ATP, citrate, $\alpha-\mathrm{KG}$ and malate concentrations in the control livers increased gradually, (suggesting increased Kreb cycle activity) but these changes did not occur in the MCS newborns. Additionally, lower Kreb cycle intermediate concentrations suggest that the activity of this energy producing pathway was attenuated in fasted MCS newborn pups (15). Significantly lower ATP levels in the MCS pups also affected the energy charge, which was reduced at 6 and $9 \mathrm{~h}$ of age. As discussed above, glycolytic flux appeared more active in the MCS pups; nevertheless, energy production through this pathway was still not sufficient to maintain hepatic ATP concentration within the control level; thus, a deficiency of oxidative substrates may have produced this attenuated energy state.
The transient episode of hypoglycemia and diminished FFA and glycerol levels noted at $3 \mathrm{~h}$ of age is thus only one manifestation of the perturbations produced by the combined substrate deficiency states produced by maternal starvation and neonatal starvation (7). Fasting hypoglycemia at $3 \mathrm{~h}$ of age may be related to enhanced tissue clearance of glucose at a time when alternate fuel availability was diminished. The rapid and marked fall of ketone bodies after birth, together with lower plasma levels of glycerol and FFA suggest that glucose oxidation is obligated to support systemic oxidative metabolism as alternate fuel availability is attenuated (17). Despite normal rates of glucose turnover, tissue extraction of glucose as an obligatory substrate results in lower glucose levels. The recovery of blood glucose levels at 6,9 , and $24 \mathrm{~h}$ may result, in part, from oxidation of alternate fuels or, more likely, from a reduction of total energy production as evident by diminished hepatic ATP, and Kreb cycle intermediate concentrations and altered hepatic energy charge in MCS pups.

\section{REFERENCES AND NOTES}

1. Atkinson, D. E. and Walton, G. M.: Adenosine triphosphate conservation in metabolic regulation. J. Biol. Chem., 242: 3239 (1967)

2. Boyd, R. D., Morris, F. H., Meschia, G., Makowski, E. L., and Battaglia, F. C.: Growth of glucose and oxygen uptaes by fetuses of fed and starved ewes. Am. J. Physiol., 225: 897 (1973).

3. Bressler, R. and Brendel, K.: The mechanism of the toxicity of 4-pentenoic acid. Trans. Assoc. Am. Phys., 82: 153 (1969).

4. Clark, M. G., Kneer, N. M., Bosch, A. L., and Lardy, H. A.: The fructose 1, 6diphosphatase-phosphofructokinase substrate cycle. J. Biol. Chem., 249: 5695 (1974).

5. Edson, J. L., Hudson, D. G., and Hull, D.: Evidence for increased fatty acid transfer across the placenta during a maternal fast in rabbits. Biol. Neonate, 27: 50 (1975)

6. El-Refai, M. and Bergman, R. N.: Simulation study of control of hepatic glycogen synthesis by glucose and insulin. Am. J. Physiol., 231: 1608 (1976).

7. Ferre, P., Pegorier, J. P., Marliss, E. B., and Girard, J. R.: Influence of exogenous fat and gluconeogenic substrates on glucose homeostasis in the newborn rat. Am. J. Physiol., 234: E129 (1978).

8. Garber, A. J. and Hanson, R. W.: The control of phosphoenolpyruvate formation by rabbit liver mitochondria. J. Biol. Chem., 246: 5555 (1971).

9. Gilbert, M. and Bourbon, J.: Effects of acute variation of fetal glycemia on glycogen storage and on glycogen synthase and phosphorylase activities in the liver of the rat fetus. Diabetes, 29: 266 (1980).

10. Girard, J. R., Chanez, C., Kervran, A., Tordet-Caridroit, C., and Assan, R.: Studies on experimental hypotrophy in the rat. III. Plasma insulin and glucagon. Biol. Neonate, 29: 262 (1976).

11. Girard, J. R., Ferre, P., Gilbert, M., Kervran, A., Assan, R., and Marliss, E. B.: Fetal metabolic response to maternal fasting in the rat. Am. J. Physiol., 232; E456 (1977).

12. Hue, L. and Hers, H. G.: Utile and futile cycles in the liver. Biochem. Biophys. Res. Commun., 58: 540 (1974).

13. Kawai, Y. and Arinze, I. J.: Activation of glycogenolysis in neonatal liver. J. Biol. Chem., 256: 853 (1981)

14. Kliegman, R. M., Miettinen, E-L., and Adam, P. A. J.: Fetal and neonatal response to maternal canine starvation: Circulating fuels and neonatal glucose production. Pediatr. Res., 15: 945 (1981).

15. Lopes-Cardozo, M. and Van Den Bergh, S. G.: Ketogenesis in isolated rat liver mitochondria. I. Relationships with the citric acid cycle and with the mitochondrial energy state. Biochem. Biophys. Acta, 283: 1 (1972).

16. Miettinen, E-L: Effect of maternal canine starvation on fetal and neonatal liver metabolism. Am. J. Physiol., 240: E86 (1981).

17. Miettinen, E-L, Kliegman, R. M., and Tserng, K-Y.: Fetal and neonatal responses to extended maternal canine starvation. I. Circulating fuels and glucose and lactate turnover. Pediatr. Res., 17: (1983).

18. Reisner, S. H., Aranda, J. V., Colle, E., Papageorgiou, A., Schiff, D., Scriver, C. R., and Stern, L.: The effect of intravenous glucagon on plasma amino acids in the newborn. Pediatr. Res., 7: 184 (1973).

19. Schwartz, A. L. and Rall, T. W.: Hormonal regulation of glycogen metabolism in human fetal liver. Diabetes, 24: 1113 (1975)

20. Seccombe, D. W., Harding, P. G. R., and Possmayer, F.: Fetal utilization of maternally derived ketone bodies for lipogenesis in the rat. Biochem. Biophys. Acta, 488: 402 (1977)

21. Shambaugh, G. E., Koehler, R. A., and Yokoo, H.: Fetal fuels. III. Ketone utilization by fetal hepatocyte. Am. J. Physiol., 235: E330 (1978).

22. Simmons, M. A., Meschia, G., Makowski, E. L., and Battaglia, F. C.: Fetal metabolic response to maternal starvation. Pediatr. Res., 8: 830 (1974)

23. Songu, E., Haugaard, E. S., Wildey, G., and Haugaard, N.: The relationship between uracil nucleotide concentrations and glycogen synthesis in hepatocytes from fed and fasted rats. Metabolism, 30: 119 (1981)

24. Sutton, R. and Pollack, J. K.: Hormone-initiated maturation of rat liver mitochondria after birth. Biochem. J., 186: 361 (1980).

25. Taylor, S. I., Mukherjee, C., and Jungas, R. L.: Regulation of pyruvate dehydrogenase in isolated rat liver mitochondria. J. Biol. Chem., 250: 2028 (1975).

26. Williamson, D. H., Lund, P., and Krebs, H. A.: The redox state of free nicotin- 
amide-adenine dinucleotide in the cytoplasm and mitochondria of rat liver. Biochem. J., 103: 514 (1967).

27. Willamson, J. R., Kreisberg, R. A., and Felts, P. W.: Mechanism for the stimulation of gluconeogenesis by fatty acids in perfused rat liver. Proc. Natl. Acad. Sci. USA, 56: 247 (1966).

28. The authors would like to express their appreciation to Mr. George Campbell, Ms. Sharon McBee and Ms. Alice Sutton for their technical assistance, and Ms. Sandra Hartman for her professional preparation of this text. We also

Copyright $(1) 1983$ International Pediatric Research Foundation, Inc $0031-3998 / 83 / 1708-0639 \$ 02.00 / 0$ express our gratitude to Dr. Sherman D. Sachs of Veterinary Medicine of Case Western Reserve University for surgical and general care of the dogs.

29. This work was supported by NICHHD grant \#HD 05740

30. Requests for reprints should be addressed to: Dr. R. M. Kliegman, Department of Pediatrics, Rainbow Babies and Childrens Hospital, 2101 Adelbert Road Cleveland,-Ohio 44106

31. Received for publication February 8, 1982.

32. Accepted for publication December 13, 1982.

Printed in U.S.A. 